Postprint of Food Microbiology Volume 59, October 2016, Pages 104-111

doi: 10.1016/j.fm.2016.05.017

\title{
Survival of foodborne pathogens in natural cracked olive brines
}

Eduardo Medina a,1, Verónica Romero-Gil a,b,1, Antonio Garrido-Fernández a \& Francisco Noé Arroyo-López ${ }^{\text {a, * }}$

a Food Biotechnology Department. Instituto de la Grasa (CSIC). University Campus Pablo de Olavide, Building 46. Ctra. Utrera, km 1. 41013 Seville (Spain).

b Regulatory Council of Protected Designation of Origen Aloreña de Málaga table olives. C/ Dehesa, 80. 29560 Pizarra, Málaga (Spain).

Running title: Survival of pathogens in olive brines

${ }^{1}$ Both authors contributed equally to this work.

*Correspondence to: Francisco Noé Arroyo López, Ph.D. e-mail: fnarroyo@cica.es 


\section{Abstract}

This work reports the survival (challenge tests) of foodborne pathogen species (Escherichia coli, Staphylococcus aureus, Listeria monocytogenes, and Salmonella enterica) in Aloreña de Málaga table olive brines. The inhibitions were fit using a loglinear model with tail implemented in GInaFIT excel software. The olive brine had a considerable inhibitory effect on the pathogens. The residual (final) populations ( $F p$ ) after 24 h was below detection limit $\left(<1.30 \log _{10} \mathrm{cfu} / \mathrm{mL}\right)$ for all species assayed. The maximum death rate $\left(k_{\max }\right)$ was $9.98,51.37,38.35$ and $53.01 \mathrm{~h}^{-1}$, while the time for 4 $\log _{10}$ reductions $\left(4 D_{r}\right)$ was $0.96,0.36,0.36$ and $0.24 \mathrm{~h}$ for E. coli, S. aureus, $L$. monocytogenes and S. enterica, respectively. Brine dilutions increased Fp and 4Dr, while decreased $k_{\max }$. A cluster analysis showed that $E$. coli had an overall quite different behaviour being the most resistant species, but the others bacteria behaved similarly, especially S. aureus and S. enterica. Partial Least Squares regression showed that the most influential phenols on microbial survival were EDA (dialdehydic form of decarboxymethyl elenolic acid), HyEDA (EDA linked to hydroxytyrosol), hydroxytyrosol 4-glucoside, tyrosol, and oleoside 11-methyl ester. Results confirm the adverse habitats of table olives for foodborne pathogenic microorganisms.

Keywords: Challenge tests; Food safety; Phenolic compounds; Survival models; Table olives. 


\section{Introduction}

Table olives are among the most important fermented vegetable of the food industry with a worldwide production above 2.4 million tons per year (IOC, 2016). They are considered as ready-to-eat products that are consumed without prior cooking. Pasteurization is frequently used to guarantee the stability and safety of fermented olives, but sometimes olives are not subjected to a heat treatment (Garrido-Fernández et al., 1997). This way, microbiota is not always removed thus pathogen contaminations is a potential public health concern. Biogenic amines, mycotoxins, and the presence of pathogens are among the most probable biological hazards found in table olives (Medina-Pradas and Arroyo-López, 2015). Thus, it is necessary to provide scientific data about foodborne pathogen presence/survival on fermented olives, especially in those processed as natural olives, which are commercialized without heat treatment and stabilized only by physicochemical conditions (Garrido-Fernández et al., 1997).

Table olives, as well as other fermented and acidified vegetable foods, have a long history of microbial safety. However, acid-resistant strains of certain pathogenic bacteria have shown that acidic foods may be a source of foodborne diseases (Centers for Disease Control and Prevention, 1996, 1999). Diverse authors have studied the survival and determined the presence of pathogenic bacteria (Escherichia coli, Listeria monocytogenes, Staphylococcus aureus and Salmonella sp.) in diverse table olive trade preparations (Spyropoulou et al., 2001; Caggia et al., 2004; Pereira et al., 2008; RASFF portal 2012a; Argyri et al., 2013; Grounta et al., 2013; Medina et al., 2013; Tataridou and Kotzekidou, 2015). Botulism, associated with Clostridium botulinum growth, is certainly the most relevant biohazard in table olives with several reported outbreaks (Debord et al., 1920; Fenicia et al., 1992; Cawthorne et al., 2005; Jalava et al., 2011; 
Pingeon et al., 2011; RASFF portal 2012b). However, the relation between the behaviour of these microorganisms in the olive environment with the presence of phenolic compounds provided by fruits has been scarcely studied. These antimicrobial compounds could limit the growth of microorganisms, mainly in non-alkali-treated olives (Medina et al., 2008, 2009, 2013; Brenes et al., 2011).

Aloreña de Málaga table olives are processed as directly brined green olives under a Spanish Protected Designation of Origin (PDO) recognized by the European Union (DOUE, 2012). For cured PDO Aloreña de Málaga processing, fruits are harvested, and directly brined in a 60-100 g/L NaCl solution. After fermentation, the olives are cracked, seasoned, and then packaged depending on demand (López-López and Garrido-Fernández, 2006). To preserve their typical green aspect and organoleptic characteristics, fruits are not stabilized by heat treatment. Therefore, safety issues such as the hygienic conditions and studies of foodborne pathogens prevalence in this table olive speciality are of particular interest for risk assessment during storage/shelf life.

The present survey aims to determine the survival of diverse foodborne pathogens (E. coli, S. aureus, L. monocytogenes, and S. enterica) in the packaging brines of ready-to-eat Aloreña de Málaga table olives. Multivariate statistical approaches were used to relate the presence of phenolic compounds to their survivals.

\section{Materials and methods}

\subsection{Preparation of olive brines}

PDO Aloreña de Málaga fruits were fermented for 3 months in an initial 60 g/L $\mathrm{NaCl}$ solution in COPUSAN S.C.A. (Alozaina, Málaga, Spain) during 2013/2014 season. Then, $10 \mathrm{~kg}$ of fruits were cracked, washed in water for $24 \mathrm{~h}$ to remove excess 
of salt, and placed in $10 \mathrm{~L}$ of $20 \mathrm{~g} / \mathrm{L}$ of $\mathrm{NaCl}$ brine at $4^{\circ} \mathrm{C}$ to obtain an equilibrium between fruits and liquid. After 14 days, brines were removed, filter-sterilized $(0.2 \mu \mathrm{m}$ pore size), and analysed to determine its composition (Table 1). Phenolic, oleosidic compounds and reducing sugars in brines were determined by HPLC according to Medina et al. (2013) and Rodríguez-Gómez et al. (2012), respectively. Salt and pH were analysed according to the protocols described by Garrido-Fernández et al. (1997).

The original olive brine (B:10/10) was then diluted with sterile distilled water to reach always a final volume of $10 \mathrm{~mL}$. The following dilutions (olive brine/sterile distilled water) were prepared: i) B:7.5/10 (7.5 mL + $2.5 \mathrm{~mL})$, ii) B:5/10 (5.0 mL + 5.0 mL), iii) B:2.5/10 (2.5 mL + 7.5 mL) and iv) B:1/10 (1.0 mL + 9.0 mL). Also, a medium with only sterile distilled water (C:0/10) was used as a control. Levels of $\mathrm{pH}$, salt, and sugar concentrations in the different dilutions and control were always kept constant by addition of $\mathrm{HCl}, \mathrm{NaCl}$, and reducing sugars (glucose, fructose, sucrose, and mannitol), respectively. Therefore, the described above media only differed in the levels of phenolic compounds, which were proportional to the dilution factors.

The ability of original olive brines to support microbial growth was also confirmed by inoculating the undiluted brine with a strain of Lactobacillus pentosus (TOMC-LAB2) and other of Lodderomyces elongisporus (TOMC-Y73), isolated previously from diverse table olive processing (Bautista-Gallego et al., 2013; RomeroGil et al., 2016) and belonging to the Table Olive Microorganisms Collection of Instituto de la Grasa (CSIC-Seville). For this purpose, the microorganisms were cultured at $30^{\circ} \mathrm{C}$ in MRS (Oxoid Ltd., Basingstoke, Hampshire, England) and YMPG (Difco, Becton and Dickinson Company, Sparks, MD, USA) broth, washed to remove 
the culture media and then inoculated in the brines an initial population ca. $5.0 \log$ CFU/mL. The growth was monitored by plating on their respective media.

\subsection{Pathogens and inoculum preparation}

Four strains of each pathogenic species (L. monocytogenes, S. aureus, E. coli and S. enterica) were obtained from the Spanish Type Culture Collection (CECT, Valencia, Spain). All of them are related with diverse human food poisoning and included in the biosafety level 2. L. monocytogenes CECT $4031^{\mathrm{T}}$ (isolated from rabbit), CECT 4032 (soft cheese), CECT 5366 (human origin) and CECT 7467 (poultry) were cultured at $37^{\circ} \mathrm{C}$ in Brain Heart Infusion (BHI, Oxoid) with or without agar (15 g/L). $S$. aureus CECT 86 ${ }^{\mathrm{T}}$ (isolated from human pleural fluid), CECT 239 (human lesion), CECT 240 (human lesion) and CECT 976 (ham); S. enterica sv. Typhimurium CECT $722^{\mathrm{T}}$ (natural environment), CECT 443 (human), CECT 4156 (chicken alimentary tract), and S. enterica sv. Enteritidis CECT 4300 (unknown); E. coli CECT 434 (clinical isolate) and the strains with serotype O157:H7 CECT 4267, CECT 4782 and CECT 5947 (isolated from human faeces) were all cultured at $37^{\circ} \mathrm{C}$ in nutrient broth prepared with 5 g/L “Lab-Lemco” powder (Oxoid), $10 \mathrm{~g} / \mathrm{L}$ of Neutralized bacteriological peptone (Oxoid), $5 \mathrm{~g} / \mathrm{L} \mathrm{NaCl}$, and $15 \mathrm{~g} / \mathrm{L}$ of agar in the case of solid medium (pH 7.2).

All these strains were maintained at $-80^{\circ} \mathrm{C}$ in the adequate culture broths with 20 g/L glycerol. Before the experiments, each strain was cultured in their respective broth media with a previous pre-adaptation phase where $\mathrm{NaCl}(15 \mathrm{~g} / \mathrm{L})$ and $\mathrm{pH}(5.5)$ were modified in order to acclimatize the microorganisms to the olive brines. Overnight cultures were centrifuged, washed and re-suspended in sterilized saline solution (9 g/L $\mathrm{NaCl}$ ). Cocktails of each pathogenic species were prepared by mixing the same quantities of the corresponding strains. The volumes were calculated to obtain ca. 8 log 
$\mathrm{cfu} / \mathrm{mL}$ of each strain as initial inoculums. An enumeration of the inoculated cells to confirm the initial population was done in duplicate, using the appropriate medium.

All treatments were dispensed into Eppendorf tubes $(1.4 \mathrm{~mL})$, added with 0.1 $\mathrm{mL}$ of each bacterial cocktail, and gently mixed. The challenge tests were performed in duplicate at room temperature $\left(20 \pm 2^{\circ} \mathrm{C}\right)$, obtaining the average of two individual inoculations. Samples from each tube were removed at different times $(0,0.5,1,3,8$ and $24 \mathrm{~h}$ ), diluted in $1.0 \mathrm{~g} / \mathrm{L}$ peptone and plated to count cultivable cells (Spiral Plater Wasp 2, Don Whitley Sci. Ltd., Shirpley, UK) in nutrient agar for S. aureus, E. coli and S. enterica and in BHI for L. monocytogenes.

\subsection{Curve fitting of the foodborne pathogen cocktails}

The responses of the different pathogen cocktails in the sterile olive brine and their dilutions were fitted using the log-linear model with a tail (Geeraerd et al., 2000), included in the GInaFIT 1.6 software excel fitting tool (Geeraerd et al., 2005). It has the following expression:

$$
\log _{10}\left(N_{t}\right)=\log _{10}\left(\left(10^{\wedge} \log _{10}(I p)-10^{\wedge} \log _{10}(F p)\right)^{*} \exp \left(-k_{\max } * t\right)+10^{\wedge} \log _{10}(F p)\right)
$$

where the parameters are: $N_{t}$, microbial population at time $t\left(\log _{10} \mathrm{cfu} / \mathrm{mL}\right) ; I p$, initial microbial population $\left(\log _{10} \mathrm{cfu} / \mathrm{mL}\right) ; \mathrm{Fp}$, final or residual population after $24 \mathrm{~h}\left(\log _{10}\right.$ $\mathrm{cfu} / \mathrm{mL})$; and $k_{\max }$, maximum death rate $\left(\mathrm{h}^{-1}\right)$. The software GInaFIT 1.6 also allows the estimation of $4 D r$ (time in hours for a reduction of $4 \log _{10}$ from $I p$ ).

\subsection{Statistical analysis}

An analysis of variance (ANOVA) was performed by means of the factorial ANOVA module of Statistica 7.1 software package (StatSoft Inc, Tulsa, OK, USA) to 
determine statistical differences among the response of the several foodborne pathogen cocktails in the original olive brine, their dilutions, and the control. Post-hoc comparisons were achieved by means of the Fisher LSD test.

Cluster analysis is a method for unsupervised learning which tries to find groups containing similar objects. In this work, it was applied based on Pearson correlation coefficient using the pathogen as grouping factor and the fitting parameters as variables. Partial Least Squares (PLS) is a technique that combines features from the Principal Component Analysis and multiple regression, which is particularly useful in case of correlated variables. Its objective is the prediction of a series of dependent variables from a set of independent variables. The prediction is based on the extraction of a series of factors (latent variables) which have the best predictive power. In this study, PLS regression was used to relate the concentration of individual phenolic compounds to the fitted parameters of the foodborne pathogens. Cluster analysis and PLS regression were performed with XLSTAT v.2015.4.01.20116 (Addinsoft, Paris, France). For the analysis, data were centred, standardised and the number of latent variables retained automatically.

\section{Results}

\subsection{Olive brines}

The physicochemical characteristics of the non-diluted olive brine are shown in Table 1. The total sugar content was $11.0 \mathrm{~g} / \mathrm{L}$, being the glucose $(6.8 \mathrm{~g} / \mathrm{L})$ and fructose $(2.1 \mathrm{~g} / \mathrm{L})$ the major components. The total phenolic compound determined by HPLC was $2.62 \mathrm{mM}$, with hydroxytyrosol $(1.04 \mathrm{mM})$ as the most relevant compound, followed by tyrosol $(0.34 \mathrm{mM})$, secologanoside $(0.31 \mathrm{mM})$, and oleoside 11-methyl 
ester (0.28 mM). Oleuropein, HyEDA, EDA and Hy-4glucoside were also detected in the brines but at lower concentrations $(0.16,0.14,0.13$ and $0.09 \mathrm{mM}$, respectively). The $\mathrm{pH}$ (5.5) and salt concentration (25 g/L) of the original brine was obtained by placing the fermented fruits in brine for 14 days at $4^{\circ} \mathrm{C}$. Since these levels are not inhibitory for the assayed microorganisms, the possible effects will then be caused exclusively by the phenolic compounds. In all the diluted brines and the control, the $\mathrm{pH}$, salt, and sugars were the same while the phenolic compounds decreased in proportion to the dilution factor.

As a preliminary test to verify the suitability of olive brines to support microbial growth, the brine was inoculated with a strain of yeast and other of lactic acid bacteria, previously isolated from packaged natural green olives and Spanish-style green table olive fermentations, respectively. Both microorganisms grew vigorously (from initial 5.0 to approximately $7.0 \log _{10} \mathrm{cfu} / \mathrm{mL}$ in $48 \mathrm{~h}$ ), as confirmed by plate count. These data supports, then, that the original Aloreña de Málaga brine is a good media for the growth of well adapted native microorganisms and inhibition is not due to the absence of fermentable substrates.

\subsection{Survival of the foodborne pathogens}

The independent inoculation of the four pathogen cocktails in the olive brines obtained from Aloreña de Málaga fruits, as well as in their respective dilutions, led to a total of 48 inhibition curves. Their changes in population were fit (Fig. 1) by the loglinear model with a tail (Geeraerd et al. 2000). In most treatments, the fit was good with an $\mathrm{R}^{2}$ above 0.95 and the Root Mean Sum of Squared Error (RMSE) ranging from 0.000 to 1.730. From the initial population (Ip), the model considers a first step of linear inactivation of microorganisms $\left(k_{\max }\right)$, followed by a second step represented by a tail or 
residual population $(F p)$. The slope of the linear inactivation phase (Fig. 1) was higher in the undiluted (B:10/10), and first two diluted brines (B:7.5/10 and B:5/10). On the contrary, pathogens kept practically constant their initial population levels $\left(\sim 8 \log _{10}\right.$ $\mathrm{cfu} / \mathrm{mL}$ ) in the control (C:0/10) through the $24 \mathrm{~h}$ study. The behaviour of E. coli cocktail in the most diluted brine (B:1/10 and $\mathrm{B}: 2.5 / 10)$ was also very similar compared to control. By contrast, for L. monocytogenes cocktail, except for the control and B:1/10 media, the response of the pathogen was very similar with small differences among the different treatments.

The inhibition parameters deduced from the fit of the mathematical model to the survival data is shown in Table 2. The Fp parameter ranged from 7.92 (E. coli in control media) to $1.30 \log _{10} \mathrm{cfu} / \mathrm{mL}$ (obtained for many of the species in the undiluted olive brine), the $k_{\max }$ parameter ranged from 0.09 (S. enterica in the control) to $53.01 \mathrm{~h}^{-1}$ (S. enterica in the undiluted olive brines), while the $4 \mathrm{Dr}$ parameter ranged from 0.24 (S. enterica in the undiluted olive brines) to $>24.0 \mathrm{~h}$ (for all assayed species in the control). The mean absolute deviations obtained for the fitted parameters from duplicate experiments were low, except for $k_{\max }$ in some treatments. In general, as olive brine was diluted, there was an increase in the final remaining population $(F p)$ and time to reduce $4 \log _{10}(4 D r)$ parameters as well as a reduction in the maximum death rate $\left(k_{\max }\right)$ value (Fig. 2). Thereby, olive brine loss its inhibitory power as dilution increased, fact that was particularly evident for dilutions higher than B:7.5/10 (Fig. 2). The factorial ANOVA analysis of the fitted parameters as a function of categorical variables (pathogen cocktails and type of medium) showed that main effects and interaction of categorical variables were statistically significant $(\mathrm{p}<0.05)$ for all parameter assayed (4Dr, $\left.k_{\max }, F p, I p\right)$. The RMSE was 1819.18, 0.259, 0.950, and 0.167 for $k_{\max }, F p, 4 D r$, and Ip parameters, respectively, indicating a poorer estimation of effects for $k_{\max }$. The 
ANOVA shows that E. coli was the most resistant microorganism, with the higher statistical significant $F p$ and $4 D r$ and the lower $k_{\max }$ values for many of the assayed conditions (Fig. 2). Otherwise, L. monocytogenes and S. enterica were the most sensible microorganisms. Furthermore, even the most diluted media (B:1/10) had a statistically significant higher inhibitory effect on parameters $4 \mathrm{Dr}$ and $\mathrm{Fp}$ compared to control (Fig. 2).

\subsection{Effect of phenolic compounds on the survival of pathogens}

The cluster analysis showed that the effect of the polyphenols on S. enterica and S. aureus in the olive brines were quite similar, although that on L. monocytogenes was close but different (Fig. 3). The response of E. coli was, on the contrary, rather different (Fig.3). The segregation is in agreement with its highest $F p$ in brine, the longest period of $4 \mathrm{Dr}$ and lowest $k_{\max }$ obtained for this species (Table 2).

The PLS regression analysis, using the concentrations of the diverse phenols as independent variables and the parameters obtained from the fitted curves ( $F p, 4 D r$, and $\left.k_{\max }\right)$ as dependent, showed that the parameters $F p$ and $4 D r$ for the $S$. aureus and E. coli cocktails were highly correlated $(>0.80)$ with all the phenolic compounds while the same parameters obtained for L. monocytogenes and S. enterica showed a sensibly lower relationship (between 0.60 and 0.70). Similarly, for all the species assayed, the parameter $k_{\max }$ was clearly related to all the phenolic compounds with values approximately similar $(>0.80)$. Therefore, the presence of phenolic compounds in the brines led to a stressing environment, which may affected the pathogens' survival selectively. 
The number of latent variables retained were 4 and the model explained a high proportion of the variable variances $\left(R^{2} X_{\text {cum }}=1.00 ; R^{2} Y_{\text {cum }}=0.92\right)$ but had a moderate overall fit $\left(\mathrm{Q}_{\text {cum }}^{2}=0.60\right)$. The contributions of the diverse phenols in the new axes (usually name as $t$ ) were the following: all the phenols contributed similarly to $t 1$ (ca. -0.300), the main contributor to $t 2$ was HyEDA (0.825), with a positive sign. In the case of $t 3$, the situation was more complex, and the phenols that most contributed to it were Hy4Glu (0.659) and EDA (-0.612). Finally, Ty (0.492) and Oleos11 (-0.644) were the main contributors to $t 4$. The projection onto new axes $t 1$ and $t 2$ (Fig. 4) show the overall correlations among the different variables and treatments. All $k_{\max }$ were strongly and positively related to the presence of phenols, mainly that of $S$. enterica which was particularly associated to HyEDA and Hy4Glu. This way, higher concentrations of phenolic compounds increased the death rate of foodborne pathogens. On the contrary, the Fp and $4 \mathrm{Dr}$ parameters were negatively linked to the presence of phenols. That is, increasing contents of phenols reduce the times to reach $4 \mathrm{Dr}$ and lead to a lower residual population of pathogens. The situation of cases (treatments) moves from left (the highest phenol content is associated to $k_{\max }$ ) to right, according to the concentrations of phenols (the control, without phenols, is associated with higher survival, $4 \mathrm{Dr}$ and Fp). Only the B:7.5/10 treatment is clearly separated from the rest.

\section{Discussion}

Predictive modelling techniques have been used in the present study to determine the survival of different foodborne pathogens in olive brine. The use of this discipline allows the quantification and comparison of the response of microorganisms in the food matrix by the use of mathematical models and estimation of fitted parameters (McMeekin et al., 1993). In this paper, we have prioritized the selection of 
strains related with human poisoning rather than a vegetable origin. Albeit strains obtained from vegetable matrixes could be better adapted to olive environment, not all of them can produce food poisoning. This way, we think that information obtained for registered pathogenic strains causing disease in humans will be more useful for further risk assessment.

Contamination of olives with pathogenic bacteria may be due to poor hygiene and unsanitary procedures by field and processing personnel, inadequate cleaning and sanitizing of processing equipment, and failure to wash the olives prior to brining. Although no severe food poisoning outbreaks have been associated with table olive consumption, recent studies have indicated the survival of certain pathogens in this fermented vegetable. Specifically, the survival of L. monocytogenes, E. coli, S. enterica and S. aureus has been reported in olive matrix (Spyropoulou et al., 2001; Skandamis and Nychas, 2003; Randazzo et al., 2012; Argyri et al., 2013; Grounta et al., 2013: Medina et al., 2013; Panagou et al., 2013). The results of many of such studies show the rapid decline of the population of these pathogens during processing indicating that the olive environment does not support well pathogen growth. Our results endorse all these previous works, and show that the assayed pathogenic bacteria were usually below the detection limits $\left(<1.3 \log _{10} \mathrm{cfu} / \mathrm{mL}\right)$ in the Aloreña de Málaga brines within the first $3 \mathrm{~h}$ after inoculation. However, in certain cases, the survival of pathogens can be extended up to 14 days even at low $\mathrm{pH}(4.2)$ and high salt concentration (60 g/L) as it was reported for L. monocytogenes, E. coli and Salmonella in the packaging of lye treated green olives of Halkidiki cv. (Argyri et al., 2013). Grounta et al. (2013) also mentioned a higher survival ( $>24 \mathrm{~h})$ of pathogenic bacteria in packaged fermented natural black olives Conservolea variety. These data show the importance of specific studies for each table olive trade preparation because of the differences in the survival among foodborne 
pathogens, as well as the considerable inhibitory effect of Aloreña de Málaga brines on pathogen survival.

Recently, Medina et al. (2013) showed that the decline of pathogen populations was variable depending on the composition of the olive brines in phenolic compounds. This way, the time needed to reduce the inoculated pathogen populations of $L$. monocytogenes, S. aureus, E. coli and S. enterica by $5 \log _{10}$ oscillated between less than 5 min and up to 17 days in the least deleterious conditions. The most lethal effect was observed in non-lye treated brines of Hojiblanca and Manzanilla variety, with a higher concentration of HyEDA and EDA phenols than the corresponding lye treated olive brines. Inhibitory compounds in table olives are formed in acid conditions by the action of endogenous enzymes of olive fruits, which hydrolyse the molecule of the oleuropein into HyEDA, and later, EDA. In contrast, these compounds are not detected in any of the brines from olives treated with $\mathrm{NaOH}$, as has already been reported (Medina et al., 2008). EDA and HyEDA, together with oleoside-11-methyl ester, are the substances in olive brines that showed the greatest antimicrobial activity (Brenes et al., 2011; Medina et al., 2009), as it was also confirmed in the present study. The curve fitting approach used in this work give us more detailed information on the pathogen responses in the olive brines (maximum death rate, residual populations, time for $4 \log _{10}$ reduction, etc.) than previous studies (Medina et al., 2013). Furthermore, the results were also subjected to a multivariate analysis for investigating the possible relationships between individual phenolic compounds and pathogen survival.

In the present study, the effect of HyEDA and EDA was noticed at lower concentrations ( $\sim 0.1 \mathrm{mM}$ for each compound) compared to values found in other table olive varieties where an inhibitory effect was obtained with values ranging from 0.4 to 
$0.8 \mathrm{mM}$ (HyEDA) and from 1.1 to $1.2 \mathrm{mM}$ (EDA) (Medina et al., 2013). A lower concentration of antimicrobial compounds requires more time of contact to reduce the initial population. Medina et al. (2009) observed that low concentrations of HyEDA $(0.075 \mathrm{mM})$ are able to reach a $4 \log _{10}$ reduction of $S$. aureus in 15 minutes. Data from this work are in line with those previous observed. In fact, the brines tested are less inhibitory when brine dilutions are between 7.5/10 and 2.5/10, which correspond to a $0.10-0.035 \mathrm{mM}$ concentration rage of HyEDA and the time needed to reach the $4 \log _{10}$ reduction is longer. Thus, HyEDA concentration found in this Aloreña de Málaga olives brine can explain by itself the bactericidal effect exerted against the pathogens tested. However an additive effect was also observed for the three antimicrobial compounds HyEDA, EDA and Ole11Me (Medina et al., 2007). From PLS regression analysis, Hy4Glu has also been correlated with the bactericidal effect exerted by the brines. However, high concentrations of this compound $(1.5 \mathrm{mM})$ did not exert any antimicrobial effect against L. pentosus (Medina et al., 2007). Nevertheless, the combined effect of all phenolic substances that may be present in olive brines is ultimately responsible for their antibacterial power (Medina et al., 2007).

In this paper, E. coli was the most resistant bacteria, while S. aureus, L. monocytogenes and S. enterica were the most sensible microorganisms. These data contrast with those obtained by Argyri et al. (2013) and Grounta et al. (2013) who found L. monocytogenes as the most resistant pathogen in the olive environment. Medina et al. (2013) studied the survival of different pathogens in various types of olive brines obtained from Hojiblanca, Manzanilla and Gordal varieties. They found that at room temperature $S$. aureus was the most resistant pathogen for all tested brines while $L$. monocytogenes and S. enterica were the least resistant to the deleterious effect of the olive brines. These differences in response of pathogenic microorganisms might be 
mainly due to: i) different origin of strains used, and ii) the different olive matrix composition (phenolic content, $\mathrm{pH}$ and salt levels, etc.). Breidt et al. (2007) have preferred the use E. coli as the target microorganisms for survival studies in pickles because its higher resistance than other foodborne pathogens, as also confirmed in the present study.

\section{Conclusions}

Aloreña de Málaga olive brines provide an adverse habitat for the development of foodborne pathogens. E. coli was the most resistant microorganisms. The results suggest its use as an appropriate target for further studies concerning olive safety issues. This species showed in less than $1 \mathrm{~h}$ a $4 \log _{10}$ reduction in its initial population, and it was below the limit of detection $\left(<1.30 \log _{10} \mathrm{cfu} / \mathrm{mL}\right)$ after $3 \mathrm{~h}$. The inhibitory power of olive brines was statistically related to the concentration of certain phenolic compounds, and it was disappearing as phenolic compounds were diluted. The pathogenic species tested could have a different survival in real olive packaging, where the presence of preservatives, lower $\mathrm{pH}$ levels and higher salt concentrations is usual. This point should be validated in further studies, also taking into account other strains of these pathogens, different physiological history of cells (e.g. acidic or low water activity adaptation) as well as the effect of different initial inoculum levels.

\section{Acknowledgements}

The research leading to these results has received funding from the Junta de Andalucía through PrediAlo Project (AGR7755: www.predialo.science.com.es) and FEDER European funds. Thanks to Copusan S.C.A (Alozaina, Málaga, Spain) for supplying the fruits and facilities for the development of experiments. FNAL wishes to 
thank the Spanish Government and CSIC for his Ramón y Cajal postdoctoral research contract, while VRG thanks the AgriFood Campus of International Excellence (ceiA3), Bank of Santander, Spanish Government and 'Aloreña de Málaga' Olive Manufacturing Association, for her pre-doctoral fellowship (training program of Ph.D. in companies). Authors also express their gratitude to F. Rodríguez-Gómez, M. Brenes, and C. Romero from Instituto de la Grasa (CSIC, Seville) for their invaluable help during HPLC analyses.

\section{References}

Argyri, A.A., Lyra, E., Panagou, E.Z., Tassou, C.C. 2013. Fate of Escherichia coli O157:H7, Salmonella Enteritidis and Listeria monocytogenes during storage of fermented green table olives in brine. Food Microbiol. 36, 1-6.

Bautista-Gallego, J., Arroyo-López, F.N., Rantsiou, K., Jiménez-Díaz, R., GarridoFernández, A., Cocolin, L. 2013. Screening of lactic acid bacteria isolated from fermented table olives with probiotic potential. Food Res. Int. 50, 135-142.

Breidt, F., Hayes, J., McFeeters, R.F. 2007. Determination of 5-log reduction times for food pathogens in acidified cucumbers during storage at 10 and $25^{\circ} \mathrm{C}$. J. Food Protect. 70, 2638-2641.

Brenes, M., García, A., De los Santos, B., Medina, E., Romero, C., Castro, A., Romero, F. 2011. Olive glutaraldehyde-like compounds against plant pathogenic bacteria and fungi. Food Chem. 125, 1262-1266.

Caggia, C., Randazzo, C.L., Salvo, M., Romeo, F., Giudici, P. 2004. Occurrence of Listeria monocytogenes in green table olives. J. Food Prot. 10, 2189-2194. 
Cawthorne, A., Celentano, L.P., D’Ancona, F., Bella, A., Masari, M., Aniballi, F., Fenicia, L., Aureli, P., Salmaso, S. 2005. Botulism and preserved green olives. Emerging Infectious Diseases 11, 781-782.

Centers for Disease Control and Prevention. 1996. Outbreak of Escherichia coli O157:H7 infections associated with drinking unpasteurized commercial apple juice - British Columbia, California, Colorado, and Washington, October 1996. Morbidity and Mortality Weekly Report, 45 (44), 975.

Centers for Disease Control and Prevention. 1999. Outbreak of Salmonella serotype Muenchen infections associated with unpasteurized orange juice - United States and Canada, June 1999. Morbidity and Mortality Weekly Report, 48(27), 582-585.

Debord, G.G., Edmondson, R.B., Thom, C. 1920. Summary of bureau of chemistry investigations of poisoning due to ripe olives. J. Ame. Medical Association 74, $1220-1221$.

DOUE. 2012. Official Journal of the European Union Regulation Nº1068/2012. L318/3

Fenicia, L., Ferrini, A.M., Aureli, P., Padovan, M.T. 1992. Epidemia di botulismo da olive nere. Industrie Alimentari 31, 307-308.

Garrido-Fernández, A., Fernández-Díez, M.J., Adams, R. 1997. Table olives. Production and processing. Chapman \& Hall, London.

Geeraerd, A.H., Herremans, C.H., Van Impe, J.F. 2000. Structural model requirements to describe microbial inactivation during a mild heat treatment. Int. J. Food Microbiol. 59, 185-209. 
Geeraerd A.H., Valdramidis, V.P., Van Impe, J.F. 2005. “GInaFiT, a freeware tool to assess non log-linear microbial survivor curves”. Int. J. Food Microbiol. 102, 95105.

Grounta, A., Nychas, G.J.E., Panagou, E.Z. 2013. Survival of food-borne pathogens on natural black table olives after post-processing contamination. Int. J. Food Microbiol. 161, 197-202.

IOC (International Olive Council). 2016. World Table Olive Figures. http://www.internationaloliveoil.org/estaticos/view/132-world-table-olive-figures. Last access: 27 January 2016.

Jalava, K., Selby, K., Pihlajasaari, A., Kolho, E., Dahlsten, E., Forss, N., Bäcklund, T., Korkeala, H., Honkanen-Buzalski, T., Hulkko, T., Derman, Y., Järvinen, A., Kotilainen, H., Kultanen, L., Ruutu, P., Lyytikaïnen, O., Lindström M. 2011. Two cases of food-borne botulism in Finland caused by conserved olives, October 2011. Euro Surveill. 2011; 16(49):pii=20034. Available online: http://www.eurosurveillance.org/ViewArticle.aspx?ArticleId=20034

López-López, A., Garrido-Fernández, A. 2006. Producción, elaboración, composición y valor nutricional de la Aceituna Aloreña de Málaga. Redagua, S.L. Pizarra (Málaga), Spain.

McMeekin, T.A., Olley, J.N., Ross, T., Ratkowsky, D.A. 1993. Predictive Microbiology: Theory and Application. John Wiley \& Sons, Inc. New York.

Medina, E., Brenes, M., Romero, C., García, A., de Castro, A. 2007. Main antimicrobial compounds in table olives. J. Agric. Food Chem. 55, 9817-9823. 
Medina, E., Romero, C., Brenes, M., García, P., de Castro, A., García, A. 2008. Profile of anti-lactic bacteria compounds during the storage of olives which are not treated with alkali. Eur. Food Res. Technol. 228, 133-138.

Medina, E., Brenes, M., García, A., Romero, C., de Castro, A. 2009. Bactericidal activity of glutaraldehyde-like compounds from olives products. J. Food Prot. 72, 2611-2614.

Medina, E., Brenes, M., Romero, C., Ramirez, E., de Castro, A. 2013. Survival of foodborne pathogenic bacteria in table olive brines. Food Control 34, 719-724.

Medina-Pradas, E., Arroyo-Lopez, F.N. 2015. Presence of toxic microbial metabolites in table olives. Front. Microbiol. 6:873.

Panagou, E.Z., Nychas, G.J.E., Sofos, J.N. 2013. Types of traditional Greek foods and their safety. Food Control 29, 32-41.

Pereira, A.P., Pereira, J.A., Bento, A., Estevinho, M.L. 2008. Microbiological characterization of table olives commercialized in Portugal in respect to safety aspects. Food Chem. Toxicol. 4, 2895-2902.

Pingeon, J.M., Vanbockstael, C., Popoff, M.R., King, L.A., Deschamps, B., Pradel, G., Dupont, H., Spanjaard, A., Houdard, A., Mazuet, C., Belaizi, B., Bourgeois, S., Lemgueres, S., Debbat, K., Courant, P., Quirin, R., Malfait, P. 2011. Two outbreaks of botulism associated with consumption of green olive paste, France, September 2011. Euro Surveill 16, (49): pii=20035. Available online: http://www.eurosurveillance.org/ViewArticle.aspx?ArticleId=20035 
Randazzo, C.L., Ribbera, A., Pitino, I., Romeo, F.V., Caggia, C. 2012. Diversity of bacterial population of table olives assessed by PCR-DGGE analysis. Food Microbiol. 32, 87-96.

RASFF Portal. 2012a. Reference number 2012.0703. https://webgate.ec.europa.eu/rasffwindow/portal.

RASFF Portal 2012b. Reference number 2012.1059. https://webgate.ec.europa.eu/rasffwindow/portal.

Rodríguez-Gómez, F., Bautista-Gallego, J., Romero-Gil, V., Arroyo-López, F.N., Garrido-Fernández, A., García-García, P. 2012. Effects of salt mixtures on Spanish green table olive fermentation performance. LWT. Food Sci. Technol. 46, 56-63.

Romero-Gil, V., Rodríguez-Gómez, F., Garrido-Fernández, A., García-García, P., Arroyo-López, F.N. 2016. Lactobacillus pentosus is the dominant species in spoilt packaged Aloreña de Málaga table olives. LWT Food Sci. Technol. 70, 252-260.

Skandamis, P.N., Nychas, G.J.E. 2003. Modeling the microbial interaction and the death of Escherichia coli O157:H7 during the fermentation of Spanish style green table olives. J. Food Protect. 66, 1166-1175.

Spyropoulou, K.E., Chorianopoulos, N.G., Skandamis, P.N., Nychas, G.-J.E. 2001. Survival of Escherichia coli O157:H7 during the fermentation of Spanish-style green table olives (conservolea variety) supplemented with different carbon sources. Int. J. Food Microbiol. 66, 3-11. 
Tataridou, M., Kotzekidou, M. 2015. Fermentation of table olives by oleuropeinolytic starter culture in reduced salt brines and inactivation of Escherichia coli O157:H7 and Listeria monocytogenes. Int. J. Food Microbiol. 208, 122-130. 


\section{Figure legends}

Figure 1. Fit of the log-linear model with tail to the experimental data of the E. coli, $S$. aureus, S. enterica and L. monocytogenes cocktails obtained in the olive brine (B:10/10), their dilutions (B:7.5/10, B:5/10, B:2.5/10, and B:1/10), and the control (C:0/10). For each sampling time, duplicated values are shown. Due to similar responses symbols may overlap. The levels of $\mathrm{pH}$ (5.5), salt (25 g/L), and sugars (11 $\mathrm{g} / \mathrm{L}$ ) were kept constant in the undiluted olive brine, diluted samples and control.

Figure 2. Graphical representation of the factorial ANOVA analysis carried out for the inhibition parameters Ip (initial population), Fp (final or residual population), 4Dr (time for $4 \log _{10}$ reduction) and $k_{\max }$ (maximum death rate) obtained with the log-linear model with tail to the survival data of the foodborne pathogens in the olive brine and their dilutions. Sa, Ec, Lm and Se stand for the S. aureus, E. coli, L. monocytogenes and S. enterica cocktails, respectively. B:10/10 stand for the undiluted olive brine, while B:7.5/10, B:5/10, B:2.5/10, B:1/10 stand for their dilutions, respectively. C:0/10 stand for the control. Vertical bars denote 0.95 confidence limits.

Figure 3. Cluster analysis grouping pathogens species according to their survival behaviours in olive brines. Sa, Ec, Lm, and Se stand for the S. aureus, E. coli, L. monocytogenes, and S. enterica cocktails, respectively.

Figure 4. PLS regression analysis. Projection of variables (independent and dependent) and treatments on the plane of the first two latent variables ( $t 1$ and $t 2$ ). The meanings of the cocktail of pathogens are: Sa, S. aureus; Ec, E. coli; Lm, L. monocytogenes and Se, $S$. enterica. Other abbreviations stands for: $F p$, final or residual population; $k_{\max }$, maximum death rate; $4 D_{r}$, time to obtain $4 \log _{10}$ reduction; Hy4Glu, Hydroxytyrosol 4glucoside; HyEDA, EDA linked to hydroxytyrosol; *, rest of phenolic compounds. Finally, B:10/10 stand for the undiluted olive brine, while B:7.5/10, B:5/10, B:2.5/10, $\mathrm{B}: 1 / 10$ stand for their different dilutions in sterile distilled water. $\mathrm{C}: 0 / 10$ is the reference for the control. 
Table 1. Physicochemical characteristics of the original Aloreña de Málaga olive brines used in the present study. Mean absolute deviations obtained from duplicated measurements in parentheses.

\begin{tabular}{lll}
\hline Parameter & Compound & Value \\
\hline Salt $(\mathrm{g} / \mathrm{l})$ & NaCl & $25.00(0.00)$ \\
$\mathrm{pH}$ & {$[\mathrm{H}+]$} & $5.50(0.01)$ \\
Sugars $(\mathrm{g} / \mathrm{l})$ & Glucose & $6.79(0.12)$ \\
& Fructose & $2.12(0.02)$ \\
& Sucrose & $0.46(0.02)$ \\
& Mannitol & $1.67(0.01)$ \\
& Total & $11.04(0.14)$ \\
Phenolic compounds $(\mathrm{mM})$ & Hy & $1.04(0.05)$ \\
& Hy 4-Glucoside & $0.09(0.01)$ \\
& Tyrosol & $0.34(0.02)$ \\
& p-coumaric acid & $0.02(0.01)$ \\
& Verbascoside & $0.02(0.01)$ \\
& HyEDA & $0.14(0.00)$ \\
& Oleuropein & $0.16(0.02)$ \\
& Comselogoside & $0.00(0.00)$ \\
& EDA & $0.13(0.00)$ \\
& Secoxyloganin & $0.08(0.01)$ \\
& Secologanoside & $0.31(0.00)$ \\
& Oleoside 11-methyl ester & $0.28(0.01)$ \\
& Total & $2.62(0.02)$ \\
\hline
\end{tabular}

Abbreviations: Hy: hydroxytyrosol. EDA: dialdehydic form of decarboxymethyl elenolic acid. HyEDA: EDA linked to hydroxytyrosol 
Table 2. Parameters obtained using the Log linear model with tail to the survival data of the foodborne pathogen cocktails in the original Aloreña de Málaga brines and their dilutions. Mean absolute deviations obtained from duplicate experiments in parentheses. Ec, Sa, $L m$ and Se stand for the E. coli, S. aureus, L. monocytones and S. enterica cocktails, respectively.

\begin{tabular}{|c|c|c|c|c|c|c|c|c|c|c|c|c|c|c|c|c|}
\hline \multirow[b]{3}{*}{ Brine* } & \multicolumn{4}{|c|}{ Initial Population (Ip) } & \multicolumn{4}{|c|}{ Final Population $(F p)$} & \multicolumn{4}{|c|}{ Time for $4 \log _{10}$ reduction (4Dr) } & \multicolumn{4}{|c|}{ Maximum death rate $\left(k_{\max }\right)$} \\
\hline & \multicolumn{4}{|c|}{$\log _{10} \mathrm{CFU} / \mathrm{mL}$} & \multicolumn{4}{|c|}{$\log _{10} \mathrm{CFU} / \mathrm{mL}$} & \multicolumn{4}{|c|}{ (h) } & \multicolumn{4}{|c|}{$\left(\mathrm{h}^{-1}\right)$} \\
\hline & Ec & $S a$ & Lm & Se & $E \boldsymbol{C}$ & Sa & Lm & Se & Ec & Sa & Lm & Se & Ec & $S a$ & Lm & Se \\
\hline $10 / 10$ & 8.10 & 8.11 & 7.64 & 7.78 & 1.60 & 1.42 & 1.34 & 1.30 & 0.96 & 0.36 & 0.36 & 0.24 & 9.98 & 51.37 & 38.35 & 53.01 \\
\hline & $(0.03)$ & $(0.00)$ & $(0.02)$ & $(0.00)$ & $(0.10)$ & $(0.03)$ & $(0.04)$ & $(0.00)$ & $(0.00)$ & $(0.12)$ & $(0.12)$ & $(0.00)$ & $(0.02)$ & $(22.57)$ & (1.68) & $(0.00)$ \\
\hline $7.5 / 10$ & 7.54 & 8.11 & 7.63 & 7.78 & 1.31 & 2.25 & 1.30 & 1.30 & 2.40 & 0.48 & 1.08 & 0.24 & 4.07 & 19.41 & 13.23 & 53.01 \\
\hline & $(0.03)$ & $(0.00)$ & $(0.02)$ & $(0.00)$ & $(0.00)$ & $(0.18)$ & $(0.00)$ & $(0.00)$ & $(0.00)$ & $(0.00)$ & $(0.60)$ & $(0.00)$ & $(0.01)$ & $(0.02)$ & (6.73) & $(0.00)$ \\
\hline $5 / 10$ & 7.35 & 8.11 & 7.65 & 7.78 & 1.82 & 2.83 & 1.30 & 1.30 & 3.12 & 0.60 & 0.48 & 0.36 & 3.10 & 18.10 & 23.27 & 40.75 \\
\hline & $(0.00)$ & $(0.00)$ & $(0.00)$ & $(0.00)$ & $(0.00)$ & $(0.03)$ & $(0.00)$ & $(0.00)$ & $(0.00)$ & $(0.12)$ & $(0.00)$ & $(0.12)$ & $(0.02)$ & $(1.85)$ & (1.01) & (12.25) \\
\hline $2.5 / 10$ & 8.25 & 8.21 & 7.40 & 7.78 & 6.94 & 3.96 & 1.68 & 1.30 & $>24.00$ & 1.32 & 0.84 & 0.48 & 2.38 & 7.87 & 13.97 & 23.28 \\
\hline & $(0.00)$ & $(0.03)$ & $(0.24)$ & $(0.00)$ & $(0.01)$ & $(0.02)$ & $(0.06)$ & $(0.00)$ & $(0.00)$ & $(0.12)$ & $(0.12)$ & $(0.00)$ & $(0.11)$ & $(0.65)$ & $(4.27)$ & (2.16) \\
\hline $1 / 10$ & 8.25 & 8.08 & 7.90 & 7.61 & 7.47 & 4.64 & 1.65 & 1.30 & $>24.00$ & $>24.00$ & 2.52 & 5.88 & 1.50 & 1.08 & 3.92 & 1.77 \\
\hline & $(0.00)$ & $(0.05)$ & $(0.07)$ & $(0.06)$ & $(0.01)$ & $(0.22)$ & $(0.15)$ & $(0.00)$ & $(0.00)$ & $(0.00)$ & $(0.12)$ & $(0.12)$ & $(0.27)$ & $(0.12)$ & $(0.27)$ & $(0.02)$ \\
\hline Control & 8.26 & 8.28 & 7.67 & 7.86 & 7.92 & 7.48 & 7.46 & 7.55 & $>24.00$ & $>24.00$ & $>24.00$ & $>24.00$ & 2.86 & 1.17 & 3.13 & 0.09 \\
\hline$(0 / 10)$ & $(0.00)$ & $(0.02)$ & $(0.02)$ & $(0.03)$ & $(0.01)$ & $(0.03)$ & $(0.03)$ & $(0.05)$ & $(0.00)$ & $(0.00)$ & $(0.00)$ & $(0.00)$ & (2.43) & $(0.00)$ & (3.13) & $(0.03)$ \\
\hline
\end{tabular}

*Proportion of the original olive brine in a final volume of $10 \mathrm{~mL}$. 


\section{Dendrogram}

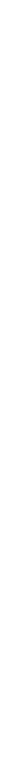


